\title{
Free vibration predicted using forced oscillation in the lock-in region
}

H. Jiao, and G. X. Wu

Citation: Physics of Fluids 30, 113601 (2018); doi: 10.1063/1.5056203

View online: https://doi.org/10.1063/1.5056203

View Table of Contents: http://aip.scitation.org/toc/phf/30/11

Published by the American Institute of Physics

\section{Articles you may be interested in}

Numerical investigation of coalescence-induced self-propelled behavior of droplets on non-wetting surfaces Physics of Fluids 30, 112102 (2018); 10.1063/1.5046056

Micro-patterning of coatings on a fiber surface exploiting the contact instabilities of thin viscoelastic films Physics of Fluids 30, 114101 (2018); 10.1063/1.5053797

Numerical study of spheres settling in Oldroyd-B fluids

Physics of Fluids 30, 113102 (2018); 10.1063/1.5032324

Dynamics of self-rewetting drop on an inclined uniformly heated substrate

Physics of Fluids 30, 112103 (2018); 10.1063/1.5050254

"Phase diagram" for viscoelastic Poiseuille flow over a wavy surface

Physics of Fluids 30, 113101 (2018); 10.1063/1.5057392

Highly accurate simplified lattice Boltzmann method

Physics of Fluids 30, 103605 (2018); 10.1063/1.5050185

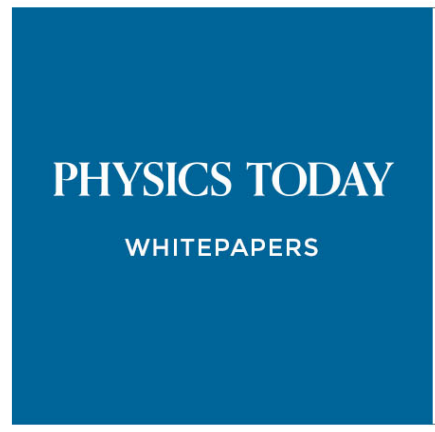

ADVANCED LIGHT CURE ADHESIVES

Take a closer look at what these environmentally friendly adhesive systems can do
READ NOW

PRESENTED BY

Q MASTERBOND 


\title{
Free vibration predicted using forced oscillation in the lock-in region
}

\author{
H. Jiao and G. X. Wu ${ }^{a)}$ \\ Department of Mechanical Engineering, University College London, London WC1E 7JE, United Kingdom
}

(Received 13 September 2018; accepted 16 October 2018; published online 5 November 2018)

\begin{abstract}
The rationale of using the results from the transverse forced oscillation of a body in the lock-in region to predict the corresponding free vibration is provided based on the mathematical analysis and verified through the numerical results at $R e=106$ obtained from the immersed boundary-lattice Boltzmann method. It is also shown through mathematical analysis that when the structural damping is fixed, if the body mass and stiffness vary together following a particular relationship, the free motion will remain the same. With this conclusion, the damping ratio is redefined using the motion frequency of the body instead of the commonly adopted natural frequency of the body. As a result, it is shown that when a cylinder is in periodic free motion, its motion will remain the same if the following two conditions are satisfied: (1) the combined mass-damping parameter remains unchanged and (2) the variations of body mass and stiffness follow a particular pattern. In this sense, the sinusoidal free motion will rely only on the combined mass-damping parameter, even at the low mass region. This is different from the previous result based on the conventionally defined mass-damping parameter. Motion amplitude and frequency contours are plotted against the damping and stiffness components based on the results of forced motion. From these, the sinusoidal free motion results can be predicted and their physics is discussed. Published by AIP Publishing. https://doi.org/10.1063/1.5056203
\end{abstract}

\section{INTRODUCTION}

Vortex-induced vibration (VIV) can be found in many fields of engineering, such as riser tubes, tethered structures in the ocean, heat exchanger tubes, and high-rise buildings. VIV may cause large-amplitude vibration of structures and lead to structural damage or even collapse of the whole system. Reviews of the earlier work were given by Bearman, ${ }^{1}$ Blevins, ${ }^{2}$ and Sumer and Fredsoe ${ }^{3}$ and the more recent ones by Williamson and Govardhan ${ }^{4}$ and Bearman. ${ }^{5}$ As a result, problems in complex fluid-structure interaction during VIV and the related problems in flow past a body in forced oscillation have become one of the fundamental subjects. There have been a large number of experimental and numerical efforts $^{6-11}$ to investigate the transverse forced oscillation of a cylinder, especially its lock-in phenomenon. Equally, there is also a large body of work ${ }^{12-19}$ on a cylinder in free motion due to the excitation of the incoming flow. However, far fewer studies have systematically considered the relationship between forced oscillation and free vibration. Thus, the present paper tries to shed some light on the rational procedure to predict the periodic free vibration of a cylinder based on its forced oscillation results in the lock-in region.

Fluid parameters which have a significant effect on both forced and free motions include the fluid density $\rho$, kinematic viscosity $v$, and incoming velocity $u_{0}$. In the forced sinusoidal oscillation of a cylinder, important parameters are the motion frequency $f_{c}$, motion amplitude $Y_{0}$, and its diameter $D$. In the free vibration, the body mass $m$, structural damping $b$, and structural stiffness $k$ become important together with $D$,

a)Email: g.wu@ucl.ac.uk while motion frequency and amplitude are to be determined instead of being prescribed. The rationale of using forced oscillation results to predict free vibration is based on energy transfer $E=\int_{0}^{T_{c}} F_{L}(t) \dot{Y}(t) d t$ from the flow to the cylinder over a period $T_{c}$. Here $F_{L}$ is the lift force of the body by the fluid and $\dot{Y}$ is the oscillation velocity of the body. In the forced oscillation, results are obtained at various prescribed motion frequencies $f_{c}$ and motion amplitudes $Y_{0}$. It is common that energy transfer contours are plotted with $f_{c}$ and $Y_{0}$ being the horizontal and vertical axes, respectively. In the free motion, body mass $m$ and structural damping $b$ are given together with structural stiffness $k$. The free motion frequency $f_{c}$ and amplitude $Y_{0}$ can be obtained from the measurement or simulations. Comparison could then be made between the energy transfer in the free motion and that on the energy transfer contours of the forced motion to see the compatibility of these two motions.

The comparison between the forced and free motions is based on the assumption of their equivalence. One should note that the forced motion is imposed by an external force. If the external force could be replaced by the corresponding mechanic mechanism, such as the structural damping $b$ and structural stiffness $k$, the forced motion is then equivalent to the free motion with such a mechanic mechanism without external force. A necessary condition for this equivalence is a positive value of $E$, which represents net energy transfer from the flow to the cylinder. Leonitini et al. ${ }^{20}$ undertook numerical studies on both forced and free motions at Reynolds number $R e=u_{0} D / v=200$ by a spectral-element technique coupled with a high-order three-step time-splitting scheme. It should be noted that $\rho, u_{0}$, and $D$ are used for nondimensionalisation and the normalized parameters are marked by $*$ below. A zero-energy contour $E^{*}=0$ on the $f_{c}^{*}-Y_{0}^{*}$ plane was 
displayed in the lock-in region of the forced oscillation within $0.14<f_{c}^{*}<0.24$. They also obtained $E^{*}$ from free vibration with a given body mass $m^{*}=m / \rho D^{2}=10$ and structural damping ratio $\zeta=b /\left[2\left(m+M_{p}\right) \omega_{n}\right]=0.01$ in the same motion frequency region as that of forced motion. Here $M_{p}=\frac{\pi}{4} \rho D^{2}$ is the potential flow added mass and $\omega_{n}=\sqrt{k /\left(m+M_{p}\right)}$ is the natural angular frequency of the body with the added mass effect. It was found that all $E^{*}$ from free vibration was in the zone of the positive energy transfer obtained from the forced oscillation, which was qualitatively consistent with the positive damping ratio $\zeta$. Kumar et al. $^{21}$ used a more stringent lock-in criterion, which was adopted to obtain energy transfer contour in lock-in forced motion at $R e=100$. The free vibration with $\zeta=0$ was considered. Its results of $f_{c}^{*}$ and $Y_{0}^{*}$ were used on the energy transfer contour of the forced motion and were found to lie almost on the $E^{*}=0$ line, which was quantitatively consistent with $\zeta=0$.

Some other studies ${ }^{22-24}$ have indicated that the results of $f_{c}$ and $Y_{0}$ from the free motion could fall into regions of negative $E$ predicted from the forced oscillation, especially at a high Reynolds number. Such a contradiction may be due to the fact that the free motion of a cylinder with constant stiffness and structural damping is in fact not fully sinusoidal as imposed in the forced oscillation. In other words, the external force in the forced sinusoidal motion cannot be equivalently replaced by the constant mechanical damping and stiffness. In general, the motion in forced oscillation can be prescribed precisely sinusoidal, while the hydrodynamic force may not be exactly sinusoidal, especially at a high Reynolds number. In such a case, exactly equivalent free motion of a cylinder with constant structural stiffness and damping is no longer possible. For example, Hover et al. ${ }^{22}$ compared some results from forced and free vibrations in the regime of $1 / 12<f_{c}^{*}$ $<1 / 3$ at $R e=3800$ and at zero energy transfer condition. The nondimensionalized mass was set as $m^{*}=\pi / 4$ and structural damping ratio was taken as $\zeta=0$ in the free motion. It was found that in the most cases the values of $Y_{0}^{*}$ and $f_{c}^{*}$ from the free motion were not on the zero energy transfer encounter line obtained from the forced motion, including the case of the largest $Y_{0}^{*}$ in the free motion. This means that the external force in the forced motion could not be exactly replaced by the mechanical system with constant damping and stiffness.

Staubli $^{25}$ and Morse and Williamson ${ }^{26}$ adopted a method different from that mentioned above to investigate the rationale of using forced oscillation results to predict free vibration. Both the maximum value of lift force $F_{L 0}$ and phase angle $\phi$ between the lift coefficient and cylinder motion can be measured from forced motion at various $f_{c}$ and $Y_{0}$ first, and then the response of possible lock-in free motions with various structural stiffnesses $k$ (or various natural frequencies of the cylinder $f_{n}$ ) at a given $m$ and $b$ can be predicted based on results from forced oscillation. Comparison can be subsequently made between the predicted free motion results and those corresponding real free motion results. Staubli ${ }^{25}$ used experimental data from forced oscillation with $Y_{0}^{*}=(0,0.8)$ and $f_{c}^{*}=(0.09,0.33)$ at $R e=60000$ to make prediction for the response of free vibration with a given $m^{*}=247.78$ and $\zeta=0.00356$ and then compared with experimental measurements of free vibration from the work of Feng. ${ }^{27}$ The prediction was reasonable for natural frequency within $f_{n}^{*}=(0.15,0.2)$ but was not close at $f_{n}^{*}=(0.12,0.15)$. It ought to be pointed out that the Reynolds number of the free-vibration experiment of Feng ${ }^{27}$ was not fixed at $R e=60000$ and therefore the conditions for the forced motion and free motions were not exactly the same. Morse and Williamson ${ }^{26}$ extended the frequency and amplitude ranges of forced oscillation to predict freevibration response at a different Reynolds number $R e=4000$. The motion amplitude was taken as $Y_{0}^{*}=(0,1.6)$ and the frequency $f_{c}^{*}=(0.0625,0.5)$. Two free vibration cases at $(1) \zeta=0$ and (2) $\zeta=0.0232$, respectively, with $m^{*}=8.24$ were predicted by the results of the forced oscillation. Comparison was then made with the corresponding real free motion data from the work of Govardhan and Williamson ${ }^{28}$ in which the Reynolds number was not always the same either. A good agreement was nevertheless found close to the peak amplitude $Y_{0 \max }^{*}$, where the Reynolds numbers of Morse and Williamson ${ }^{26}$ and Govardhan and Williamson ${ }^{28}$ were matched.

In the present work, we shall first provide the mathematical analysis for the conditions of the equivalent forced and free motions. This will be further confirmed by the numerical results at $R e=106$ obtained from the forced and free motion simulations. We then show that if the body mass and stiffness vary following a particular pattern, the free motion of the body will remain the same. This can be linked to the extensive studies on the effect of $m^{*} \zeta \cdot{ }^{29,30}$ It has been well established that for $O\left(m^{*}\right)=100$ and $O(\zeta)=0.001$ (or $m^{*} \zeta>0.1$ ), the amplitude at resonance is usually the largest. In such a case, the largest motion becomes a unique function of the combined $m^{*} \zeta$ parameter at resonance or $\left(m^{*}+M_{p}^{*}\right) \zeta$ in the case where the added mass is also important. The logic for this ${ }^{1,31}$ can be shown that the motion amplitude can be obtained as

$$
Y_{0}^{*}=\frac{C_{L 0} \sin \phi}{16 \pi^{2}\left(m^{*}+M_{p}^{*}\right) \zeta}\left(\frac{U^{*}}{f^{*}}\right)^{2} f^{*} .
$$

Here $U^{*}=1 / f_{n}^{*}$ is the reduced velocity. $f^{*}$ is the ratio between body motion frequency $f_{c}^{*}$ and its natural frequency $f_{n}^{*}$

$$
f^{*}=\frac{f_{c}^{*}}{f_{n}^{*}}=\sqrt{\frac{m^{*}+M_{p}^{*}}{m^{*}+M_{e}^{*}}},
$$

where $M_{e}^{*}$ is the effective added mass due to the lift force in phase with body acceleration

$$
M_{e}^{*}=\frac{C_{L 0} \cos \phi}{8 \pi^{2} Y_{0}^{*}}\left(\frac{U^{*}}{f^{*}}\right)^{2} .
$$

At resonance, $f^{*}=1$, and thus the amplitude can be written as

$$
Y_{0}^{*} \propto \frac{C_{L 0} \sin \phi}{\left(m^{*}+M_{p}^{*}\right) \zeta}
$$

which depends on $\left(m^{*}+M_{p}^{*}\right) \zeta$.

For $O\left(m^{*}\right)=1-10$ and $O(\zeta)=0.001$ (or $m^{*} \zeta \ll 0.1$ ), the large response, including the peak response, may not always be found at resonance, but at a different frequency of the lock-in region, where the body oscillation frequency matches with that of the periodic wake vortex mode. ${ }^{31,32}$ In the region of lock-in, $f^{*}$ may be no longer equal to one. For instance, $f^{*}$ was about 1.4 for the largest free motion with $m^{*}=2.4$ and $\zeta=0.0045$ in 
the lock-in region. ${ }^{4}$ From the result, it has been suggested that the amplitude is no longer a unique function of $\left(m^{*}+M_{p}^{*}\right) \zeta$, as $f^{*}=1$ in the case of large $m^{*}$. The dynamic response of the free motion would be affected by $m^{*}+M_{p}^{*}$ and $\zeta$ independently, not just by $\left(m^{*}+M_{p}^{*}\right) \zeta$ as a single parameter. ${ }^{33}$ This has led to a conclusion seemingly contradicting to the one mentioned previously. The fact is most earlier studies might have focused on the high mass $\left(O\left(m^{*}\right)=100\right)$ in aerodynamic applications where the fluid is air, not the low mass $\left(O\left(m^{*}\right)=1-10\right)$ in hydrodynamic fields where water is the fluid medium. For the high $m^{*}$, the difference between $M_{e}^{*}$ and $M_{p}^{*}$ can be neglected in $f^{*}=\sqrt{\frac{m^{*}+M_{p}^{*}}{m^{*}+M_{e}^{*}}}$, compared with $m^{*}$ in the lock-in free motion. Therefore, $f^{*}$ is very close to unity and may be regarded as 1 approximately in the full lock-in region. Thus, the largest motion occurs always at $f^{*}$ close to 1 . For the low $m^{*}$, the effect of the difference between $M_{e}^{*}$ and $M_{p}^{*}$ is relatively more significant in the lock-in free motion, and thus $f^{*}$ may not be close to unity in most parts of the lock-in region, including that at the peak response. It should also be noted that most previous studies on the effect of $m^{*} \zeta^{1,4}$ were based on experimental results, in which structural parameters $(m, k, b$, and $D)$ and the fluid medium ( $\rho$ and $v)$ were fixed, and the incoming fluid velocity $u_{0}$ was varied. It means that $U^{*}$ or $f_{n}^{*}$ through the lock-in region changed with various velocity $u_{0}$, not with $k$ or $f_{n}$. In these experiments, the effect on the results was attributed to the variation of $U^{*}$ (or $f_{n}^{*}$ ) rather than the Reynolds number. ${ }^{5,28}$

Here once it is demonstrated that it is possible to have the same free motion response of a cylinder when the structural damping $b$ is fixed, while the body mass $m$ and stiffness $k$ can be different, we redefine the damping ratio as $\zeta_{c}=b / 2\left(m+M_{p}\right) \omega_{c}$. Here the motion frequency of the body $\omega_{c}$ is used instead of the natural frequency of the body $\omega_{n}$. Using this definition, we are then able to conclude that when a sinusoidal free motion of a cylinder is achieved, its motion will remain the same if (1) the mass-damping $\left(m+M_{p}\right) \zeta_{c}$ remains unchanged and (2) the variation of $m+M_{p}$ and that of the stiffness $k$ follow a particular relationship. In this sense, even away from the resonance, for different $m+M_{p}$, whether it is high or low, the motion of the body will always remain the same, provided the above conditions are met. In other words, the motion will depend on mass-damping $\left(m+M_{p}\right) \zeta_{c}$, even when the individual $m+M_{p}$ and $\zeta_{c}$ change.

In the following sections, the governing equations and the numerical method based on the immersed boundary-lattice Boltzmann method (LBM) are first introduced in Sec. II. This is followed by the mathematical analysis for the condition of equivalence between the forced and free motions in Sec. III. Results are provided in Sec. IV, followed by conclusions in Sec. V.

\section{GOVERNING EQUATIONS AND NUMERICAL METHOD}

\section{A. Immersed boundary-lattice Boltzmann method}

The flow of viscous, incompressible, and Newtonian fluid is governed by the following continuity equation and NavierStokes (NS) equation,

$$
\begin{gathered}
\nabla \cdot \vec{u}=0, \\
\frac{\partial \vec{u}}{\partial t}+(\vec{u} \cdot \nabla) \vec{u}=-\frac{\nabla p}{\rho}+v \nabla^{2} \vec{u},
\end{gathered}
$$

where $\vec{u}$ is the fluid velocity, $p$ is the pressure, $\rho$ is the fluid density, and $v$ is the kinematic viscosity. These two equations are then combined with the no-slip condition on the solid surface $s$ or

$$
\vec{u}=\vec{U}^{d}(s),
$$

where $\vec{U}^{d}$ is the velocity of the body surface.

\section{Immersed boundary method}

The present work is based on the lattice Boltzmann method (LBM) for governing equation in the volume coupled with immersed boundary method (IBM) conditions on the boundary. The detailed process adopted here can be found in the work of Jiao and Wu. ${ }^{34}$ IBM has been used extensively to study the interaction between the structure and fluid flow, and a latest application is, for example, that on the fluid-solid "sharp" interface. ${ }^{35}$ IBM treats structure-fluid boundary by replacing the body surface with a layer of distributed force $\vec{g}$ into Eq. (6) ${ }^{36}$ or

$$
\frac{\partial \vec{u}}{\partial t}+(\vec{u} \cdot \nabla) \vec{u}=-\frac{\nabla p}{\rho}+v \nabla^{2} \vec{u}+\vec{g} .
$$

The value of the external force $\vec{g}$ is obtained by the delta function $\delta_{h}$,

$$
\vec{g}(\vec{x}, t)=\sum_{s} \vec{G}(s, t) \delta_{h}(\vec{x}-\vec{X}(s, t)),
$$

where $\vec{x}$ is the position vector in the Cartesian coordinate system $O x y$ and $\vec{X}(s, t)$ is the position of the body surface and will change with time when the body is in motion. The required body force on the solid boundary is to ensure the no-slip condition through the proper choice of the forcing term, which is given as

$$
\vec{G}(s, t)=\frac{\vec{U}^{d}(s, t)-\vec{U}^{*}(s, t)}{\Delta t} .
$$

Here $\vec{U}^{*}$ is the velocity on the boundary without the forcing term. It is obtained from

$$
\vec{U}^{*}(s, t)=\sum_{\vec{x}} \vec{u}^{*}(\vec{x}, t) \delta_{h}(\vec{x}-\vec{X}(s, t)),
$$

where $\vec{u}^{*}$ is the fluid velocity without the forcing term from Eq. (6). Based on the work of Peskin, ${ }^{37}$ the delta function $\delta_{h}(\vec{x})$ can be written as follows:

$$
\delta_{h}(\vec{x})=\delta_{h}(x) \delta_{h}(y),
$$

where

$$
\delta_{h}(r)= \begin{cases}\frac{1}{4 h}\left[1+\cos \left(\frac{\pi|r|}{2 h}\right)\right], & |r| \leq 2 h \\ 0, & \text { otherwise }\end{cases}
$$

where $h$ is the grid size of the fluid domain. 


\section{Lattice Boltzmann method}

LBM is based on microscopic models and mesoscopic kinetic equations. ${ }^{38}$ It can recover incompressible continuity and NS equations based on the Chapman-Enskog expansion at an error proportional to $M^{2}$, where $M=u / c_{s}$ is the Mach number and $c_{s}$ is the chosen sound speed. ${ }^{39}$ To describe Eqs. (5) and (8), the LBM governing equation can be written as

$$
\begin{aligned}
f_{i}\left(\vec{x}+\vec{e}_{i} \Delta t, t+\Delta t\right)= & f_{i}(\vec{x}, t)-\frac{1}{\tau}\left[f_{i}(\vec{x}, t)-f_{i}^{e q}(\vec{x}, t)\right] \\
& +\Delta t \frac{\omega_{i} \rho}{c_{s}^{2}} \vec{e}_{i} \cdot \vec{g},
\end{aligned}
$$

where $f_{i}$ is the weighted density distribution function corresponding to each discretized velocity $\vec{e}_{i}$ and $f_{i}^{e q}$ is the corresponding equilibrium distribution function. $\tau$ in Eq. (10) is the relaxation time, and $\Delta t$ is the time step.

For the two-dimension problem, we adopt the ninediscretized velocity or D2Q9 model. ${ }^{40}$ Corresponding velocities are $\vec{e}_{i}=c(\cos ((i-1) \pi / 2), \sin ((i-1) \pi / 2)) i=1-4, \vec{e}_{i}$ $=\sqrt{2} c(\cos ((i-5) \pi / 2+\pi / 4), \sin ((i-5) \pi / 2+\pi / 4)) i=5-8$, and $\vec{e}_{0}=(0,0)$. The equilibrium distribution function is of the form

$$
f_{i}^{e q}(\vec{x}, t)=\rho \omega_{i}\left[1+\frac{\vec{e}_{i} \cdot \vec{u}}{c_{s}^{2}}+\frac{\left(\vec{e}_{i} \cdot \vec{u}\right)^{2}}{2 c_{s}^{4}}-\frac{\vec{u} \cdot \vec{u}}{2 c_{s}^{2}}\right],
$$

where weighting coefficient $\omega_{i}$ are given as $\omega_{0}=4 / 9, \omega_{i}=1 / 9$ for $i=1-4$, and $\omega_{i}=1 / 36$ for $i=5-8$. The fluid domain is then discretized by the structured mesh with $\Delta x=\Delta y=c \Delta t=h$, where $c=\sqrt{3} c_{s}$ is the lattice speed. The kinematic viscosity is $v=(\tau-0.5) c_{s}^{2} \Delta t$. The fluid density and the fluid velocity at each point can be, respectively, calculated as follows:

$$
\rho=\sum_{i=0}^{8} f_{i} \quad \text { and } \rho \vec{u}=\sum_{i=0}^{8} f_{i} \vec{e}_{i} .
$$

The pressure $p$ can be calculated by $p=\rho c_{s}^{2}$.

\section{B. Fluid force and free motion of a body}

The fluid force on the cylinder is calculated by integrating the external force $\vec{g}(\vec{x}, t)=\left(g_{x}(\vec{x}, t), g_{y}(\vec{x}, t)\right)$ over the whole fluid domain. The drag and lift forces are given by

$$
F_{D}=\iint g_{x}(\vec{x}, t) d x d y
$$

and

$$
F_{L}=\iint g_{y}(\vec{x}, t) d x d y .
$$

In reality, this integration needs to be performed only over the layer next to the body surface because of the delta function in Eq. (9). The corresponding coefficients are defined by $C_{D}=F_{D} / 0.5 \rho u_{0}^{2} D$ and $C_{L}=F_{L} / 0.5 \rho u_{0}^{2} D$, respectively.

In many engineering problems, the transverse motion of the body or the motion in the $y$ direction is the main concern because the lift (transverse) fluctuation is generally much larger than drag (in-line) fluctuation. If the body mass is $m$, the structural damping is $b$, and the stiffness is $k$, its governing equation is

$$
m \ddot{Y}+b \dot{Y}+k Y=F_{L},
$$

where $Y$ is the displacement and the overdot denotes the temporal derivative.

\section{EQUIVALENCE BETWEEN FREE MOTION AND FORCED MOTION AT LOCK-IN}

Kumar et al. ${ }^{21}$ reviewed the various criteria for lock-in at forced oscillation and proposed a more stringent criterion to describe lock-in, together with transition and no lock-in regions. The system can be regarded as the state of the lock-in when (a) the dominant frequency in the power spectrum of the lift coefficient is equal to the forced oscillation frequency $f_{c}$ and (b) other components in its power spectrum, if any, are only at integer multiples of $f_{c}$. When only the first condition is satisfied, which means that the other components of the power spectrum can be random, the region is referred to as transition one. All other situations correspond to a no lock-in region. The present work applies their criterion to identify the lock-in state.

Based on the definition of lock-in above, the oscillation of the lift force $F_{L}(t)$ should have a series of frequency components, $(n+1) f_{c}(n=0,1,2, \ldots)$, or

$$
F_{L}(t)=\sum_{n=0}^{\infty} F_{n} \sin \left[2 \pi(n+1) f_{c} t+\phi_{n}\right]
$$

In the forced motion, the external force $F_{e}$ applied on the body must follow

$$
F_{e}(t)=m \ddot{Y}(t)-F_{L}(t)
$$

Here if the mechanic system of the cylinder in free motion could provide this external force, its oscillation would be the same as that of the forced motion. In general, this requirement would not always be practical. However, as the dominant frequency is $f_{c}$, which means $F_{0}$ is much larger than all other components, it can be assumed that $F_{L}(t)$ can be approximated by the first term. In such a case, we may have

$$
\begin{gathered}
F_{L}=F_{0} \sin \left(\omega_{c} t+\phi\right), \\
Y=Y_{0} \sin \left(\omega_{c} t\right),
\end{gathered}
$$

where $\omega_{c}=2 \pi f_{c}$ is the angular frequency, $\phi=\phi_{0}$ is the phase angle between the lift force $F_{L}$ and the displacement $Y$ of the cylinder.

The external force $F_{e}$ in such a case can then be replaced by a constant stiffness $k$ and structural damping $b$ or

$$
F_{e}(t)=-k Y(t)-b \dot{Y}(t),
$$

where

$$
\begin{gathered}
k=m \omega_{c}^{2}+\tilde{k}, \\
b=\frac{F_{0} \sin \phi}{\omega_{c} Y_{0}}, \\
\tilde{k}=\frac{F_{0} \cos \phi}{Y_{0}} .
\end{gathered}
$$

The free motion is then expected to be the same as the forced motion. Based on this principle, we may use the result in the forced lock-in motion to predict the behavior of the free motion of the cylinder. It is interesting to see from the above two equations that while the choice of $b$ from Eq. (20) is fixed at given $\omega_{c}$ and $Y_{0}$, the choice of $k$ in Eq. (19) is not unique and it depends on the mass of the cylinder $m$. In other words, 


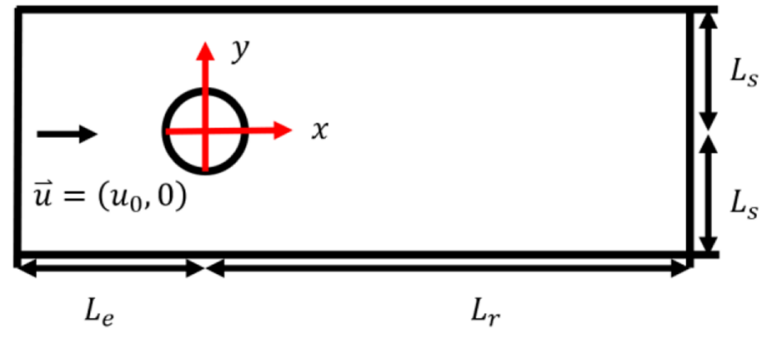

FIG. 1. Configuration of the computational domain.

the masses and stiffnesses of two cylinders may be different. Provided Eq. (19) or $m_{2}-m_{1}=\left(k_{2}-k_{1}\right) / \omega_{c}^{2}$ is satisfied in these cases, their free motions will be the same. A similar result was observed by Shiels et al. ${ }^{41}$ at $b=0$.

\section{RESULTS}

A sketch of the computational domain for forced and free motions of a circular cylinder with diameter $D$ is shown in Fig. 1. The incoming flow is from the left hand side of the body. The cylinder is located in the flow field. The computational domain is taken as $L \mathrm{e}=14 D, L \mathrm{~s}=20 D$, and $L \mathrm{r}=25.5 D$, which is chosen similar to that used by Prasanth and Mittal. ${ }^{15}$

A Dirichlet boundary condition $\left(\vec{u}=\left(u_{0}, 0\right)\right)$ is adopted at the inflow and far-field boundaries. The uniform steady flow is used in the initial condition. Nondimensionalized variables used in the subsequent paper are shown in Table I. The equation of free motion can be written as

$$
m^{*} \ddot{Y}^{*}+b^{*} \dot{Y}^{*}+k^{*} Y^{*}=C_{L} / 2,
$$

where $m^{*}=m / \rho D^{2}, b^{*}=b / \rho u_{0} D$, and $k^{*}=k / \rho u_{0}^{2}$ are the nondimensionalized body mass, damping, and stiffness, respectively. This is similar to what was adopted by Shiels et $a{ }^{41}$ It should be noted that the way to nondimensionalize the equation of free motion is not unique and other forms of equations have also been used, some of which have been discussed in the Introduction.
TABLE II. Mean drag coefficient, maximum lift coefficient, and phase angle for forced oscillation with $f_{c}^{*}=0.177$ and $Y_{0}^{*}=0.6$ at $R e=106$.

\begin{tabular}{lcccc}
\hline \hline Grid parameter & Mach number & $\overline{C_{D}}$ & $C_{L 0}$ & $\phi(\mathrm{deg})$ \\
\hline$s=70$ & $M=0.02$ & 1.801 & 1.133 & -11.4 \\
$s=105$ & $M=0.02$ & 1.810 & 1.134 & -11.4 \\
$s=70$ & $M=0.01$ & 1.800 & 1.140 & -11.4 \\
\hline \hline
\end{tabular}

\section{A. Convergence study and comparison}

Forced $\left(f_{\mathrm{c}}^{*}=0.177\right.$ and $\left.\mathrm{Y}_{0}^{*}=0.6\right)$ and free $\left(\mathrm{m}^{*}=1-2\right.$, $\mathrm{b}^{*}=0$, and $\mathrm{k}^{*}=0-10$ ) oscillations have been carried out to verify the grid convergence and the artificial compressibility of the numerical method. Convergence should be undertaken with respect to the grid size $\Delta x$, the artificial compressibility through the Mach number $M$, and the time step $\Delta t$. In the LBM method outlined above, at a given Reynolds number, the time step will be fixed when the grid parameter $s=D / \Delta x$ and Mach number have been chosen. Thus, its convergence is reflected in the convergence with $s$ and $M$, respectively.

\section{Effect of grid size}

The grid convergence is first investigated by forced and free motions through $s$. The Mach number is taken as $M=0.02$ and the Reynolds number is taken as $R e=106$. Table II shows the mean drag coefficient $\overline{C_{D}}$, maximum lift coefficient $C_{L 0}$, and phase angle $\phi$ obtained from forced oscillation with two different values of grid parameters $s=70$ and 105. Table III shows results from free vibration with $s=70$ and 105 . The largest relative error is about $0.5 \%$. Thus, in the subsequent calculations, $s=70$ is used. It should be pointed out that Eq. (22) can be calculated by the Euler method, once the lift coefficient is known from the flow field calculated by LBM coupling with IBM.

\section{Effect of artificial compressibility}

Computations are carried out for two different values of Mach numbers, $M=0.01$ and 0.02 , to study its effect

TABLE I. Nondimensionalized parameters.

\begin{tabular}{llll}
\hline \hline Body mass & $\mathrm{m}^{*}=\frac{\mathrm{m}}{\rho \mathrm{D}^{2}}$ & Reduced velocity & $\mathrm{U}^{*}=\frac{1}{\mathrm{f}_{\mathrm{n}}^{*}}$ \\
Potential flow added mass & $\mathrm{M}_{\mathrm{p}}^{*}=\frac{\mathrm{M}_{\mathrm{p}}}{\rho \mathrm{D}^{2}}$ & Wavelength & $\lambda^{*}=\frac{1}{\mathrm{f}_{\mathrm{c}}^{*}}$ \\
Structural damping & $\mathrm{b}^{*}=\frac{\mathrm{b}}{\rho \mathrm{u}_{0} \mathrm{D}}$ & Motion amplitude & $\mathrm{Y}_{0}^{*}=\frac{\mathrm{Y}_{0}}{\mathrm{D}}$ \\
Damping ratio & $\zeta=\frac{\mathrm{b}}{2\left(\mathrm{~m}+\mathrm{M}_{\mathrm{p}}\right) \omega_{\mathrm{n}}}$ & Lift coefficient & $\mathrm{C}_{\mathrm{L}}=\frac{\mathrm{F}_{\mathrm{L}}}{0.5 \mathrm{\rho u}_{0}^{2} \mathrm{D}}$ \\
Redefined damping ratio & $\zeta_{\mathrm{c}}=\frac{\mathrm{b}}{2\left(\mathrm{~m}+\mathrm{M}_{\mathrm{p}}\right) \omega_{\mathrm{c}}}$ & Amplitude of the lift coefficient & $\mathrm{C}_{\mathrm{L} 0}=\frac{\mathrm{F}_{0}}{0.5 \mathrm{u}_{0}^{2} \mathrm{D}}$ \\
Structural stiffness & $\mathrm{k}^{*}=\frac{\mathrm{k}}{\rho \mathrm{u}_{0}^{2}}$ & Drag coefficient & $\mathrm{C}_{\mathrm{D}}=\frac{\mathrm{F}_{\mathrm{D}}}{0.5 \rho \mathrm{u}_{0}^{2} \mathrm{D}}$ \\
Related stiffness & $\tilde{\mathrm{k}}^{*}=\frac{\tilde{\mathrm{k}}}{\rho \mathrm{u}_{0}^{2}}$ & Effective added mass & $\mathrm{M}_{\mathrm{e}}^{*}=\frac{\mathrm{C}_{\mathrm{L} 0} \cos \phi}{8 \pi^{2} \mathrm{Y}_{0}^{*}}\left(\frac{\mathrm{U}^{*}}{\mathrm{f}^{*}}\right)^{2}$ \\
Natural frequency of body & $\mathrm{f}_{\mathrm{n}}^{*}=\frac{\mathrm{f}_{\mathrm{n}} \mathrm{D}}{\mathrm{u}_{0}}$ & Vorticity & $\mathrm{W}^{*}=\frac{\mathrm{wD}_{\mathrm{D}}}{\mathrm{u}_{0}}$ \\
Motion frequency & $\mathrm{f}_{\mathrm{c}}^{*}=\frac{\mathrm{f}_{\mathrm{c}} \mathrm{D}}{\mathrm{u}_{0}}$ & Reynolds number & $\mathrm{Re}=\frac{\mathrm{u}_{0} \mathrm{D}}{\mathrm{v}}$ \\
Vortex frequency of a fixed cylinder & $\mathrm{f}_{\mathrm{v}}^{*}=\frac{\mathrm{f}_{\mathrm{v}} \mathrm{D}}{\mathrm{u}_{0}}$ & Grid parameter & $\mathrm{s}=\frac{\mathrm{D}}{\Delta \mathrm{x}}$ \\
Frequency ratio & $\mathrm{f}^{*}=\frac{\mathrm{f}_{\mathrm{c}}^{*}}{\mathrm{f}_{\mathrm{n}}^{*}}$ & Mach number & $\mathrm{M}=\frac{\mathrm{u}_{0}}{\mathrm{c}_{\mathrm{s}}}$ \\
\hline \hline
\end{tabular}


TABLE III. Mean drag and maximum lift coefficients, and amplitude for free oscillation with $m^{*}=1, b^{*}=0$, and $k^{*}=2.438$ at $R e=106$.

\begin{tabular}{lccccc}
\hline \hline Grid parameter & Mach number & $\overline{C_{D}}$ & $C_{L 0}$ & $f_{c}^{*}$ & $Y_{0}^{*}$ \\
\hline$s=70$ & $M=0.02$ & 1.686 & 1.065 & 0.186 & 0.515 \\
$s=105$ & $M=0.02$ & 1.688 & 1.064 & 0.186 & 0.514 \\
$s=70$ & $M=0.01$ & 1.687 & 1.070 & 0.186 & 0.518 \\
\hline \hline
\end{tabular}

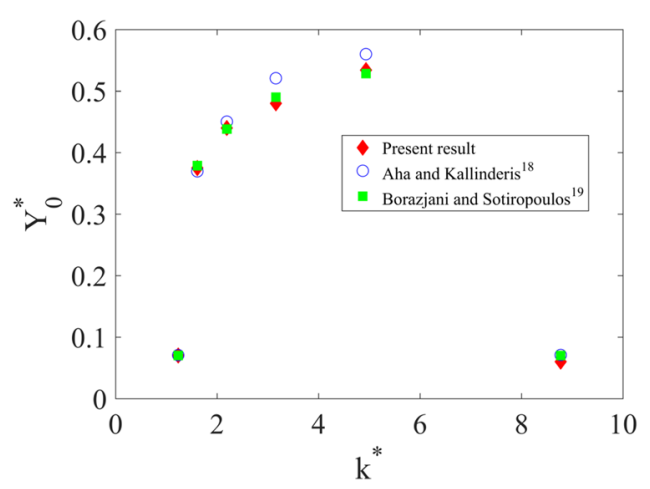

FIG. 2. Comparison of motion amplitude against $k^{*}$ for free vibration at $m^{*}=2, b^{*}=0$, and $R e=150(s=70 ; M=0.02)$.

on forced and free motions at $R e=106$. The results are shown in Tables II and III. The largest relative error is about $0.6 \%$. Thus, when $M \leq 0.02$, the effect of the Mach number can be neglected for forced and free motions. Figure 2 provides the comparison between the present results of free vibration and previous computational data from the work of Aha and Kallinderis ${ }^{18}$ and Borazjani and Sotiropoulos ${ }^{19}$ for the range of $k^{*}=0-10$ at $m^{*}=2, b^{*}=0$, and $R e=150$. There is an excellent agreement between the present and other results, suggesting that the present numerical method is accurate and resolution is adequate. In the remainder of the paper, results are computed with $s=70, M=0.02$, and $R e=106$.

\section{B. Simulations of forced motion at lock-in}

From the analysis in Sec. III, it can be seen that the free motion of a cylinder may be predicted by the results of its corresponding lock-in forced motions under certain conditions. Here we run simulations of forced motions with different amplitudes $Y_{0}^{*}=0.05-0.6$ at a given oscillation frequency $f_{c}^{*}=0.2-0.15$. We notice that both $C_{L 0}$ and $\phi$ change with $Y_{0}^{*}$. Based on our results, it is found that the frequency of the lift coefficient on a fixed cylinder is $f_{v}^{*}=0.177$ at $\operatorname{Re}=106$.

We first choose $f_{c}^{*}=0.2>f_{v}^{*}$, and simulations have been undertaken for amplitude in the range of $Y_{0}^{*}=(0.2,0.6)$. It is found that there is no lock-in when $Y_{0}^{*}<0.25$, and in the lockin region, when $Y_{0}^{*}>0.556$, the energy transfer from the fluid to the cylinder is negative. Figure 3 shows the variation of (a) $C_{L 0}$, (b) $\phi$, (c) $b^{*}$, and (d) $\tilde{k}^{*}$ with $Y_{0}^{*}$, in which $\tilde{k}^{*}$ and $b^{*}$ are related to $k$ and $b$ in Eqs. (19) and (20), respectively. Within the range of $Y_{0}^{*}=(0.25,0.556)$, the variation of the phase angle $\phi$ is from $14^{\circ}$ to $0^{\circ}$. At this oscillation frequency $f_{c}^{*}=0.2$, $Y_{0}^{*}=0.556$ is the maximum amplitude of cylinder motion, at which the equivalent free motion is possible. Beyond this value, the energy transfer from the fluid to the body is negative, which means that the corresponding structural damping would have to be negative. $Y_{0}^{*}=0.25$ is the minimum possible value for lock-in of the forced motion to occur. It is interesting to see that at $Y_{0}^{*}=0.25$ lock-in starts with the phase angle, $\phi \approx 0^{\circ}$, which is similar to that at $Y_{0}^{*}=0.556$. The values of $C_{L 0}$ at these two amplitudes are also very close. In addition, within $Y_{0}^{*}=(0.25,0.556), \tilde{k}^{*}$ decreases monotonically with the increase in $Y_{0}^{*}$.

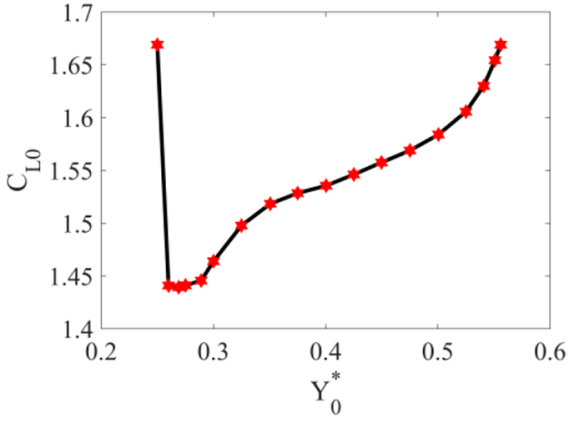

(a)

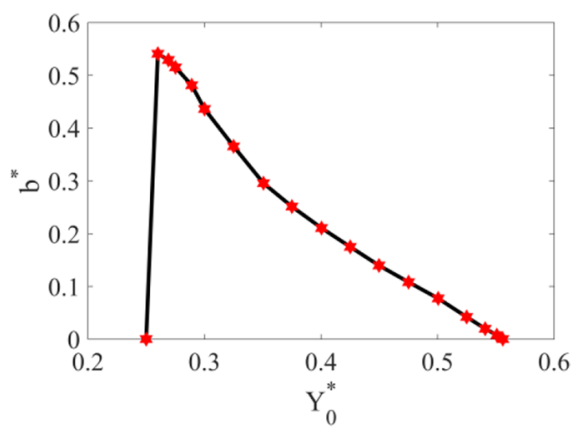

(c)

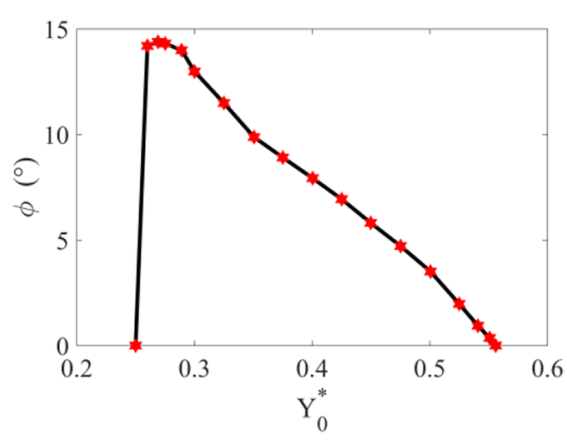

(b)

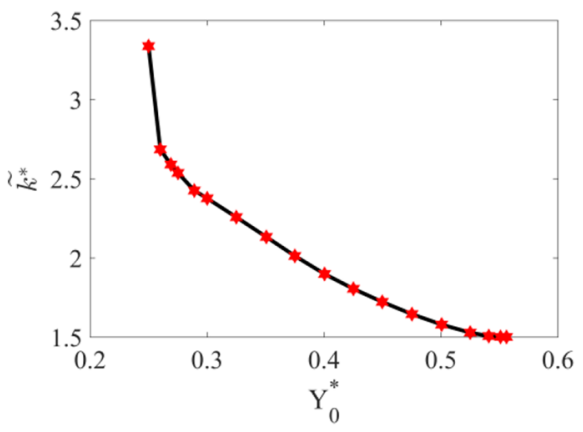

(d)
FIG. 3. Results of lock-in forced oscillation with different amplitudes at $f_{c}^{*}=0.2$. 


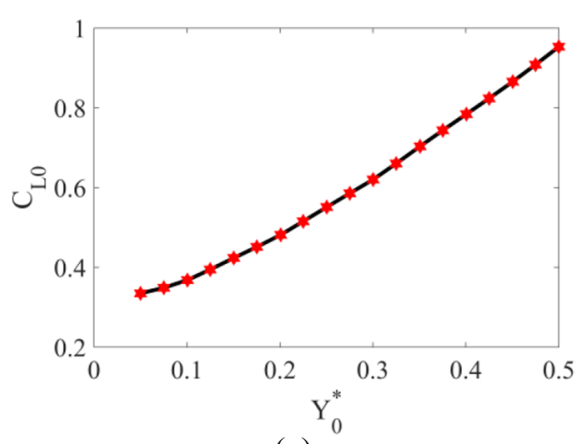

(a)

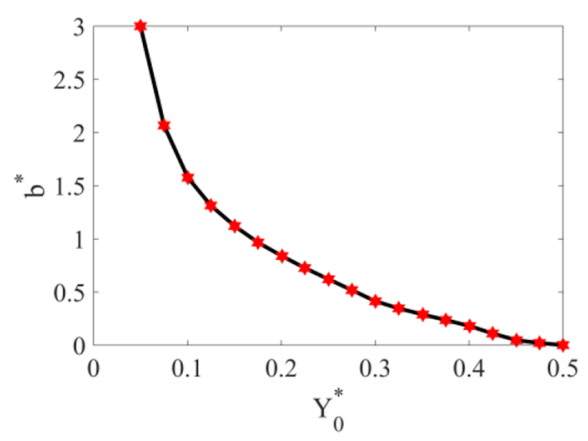

(c)

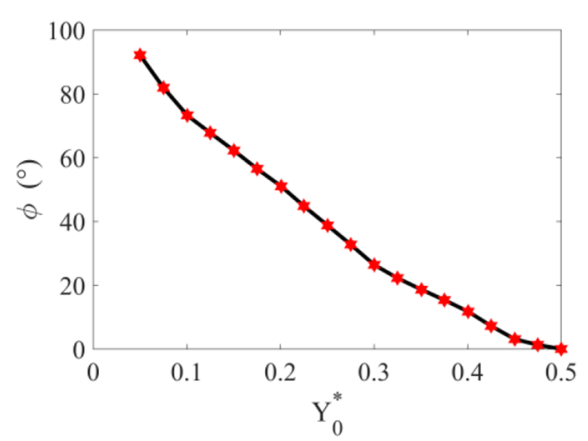

(b)

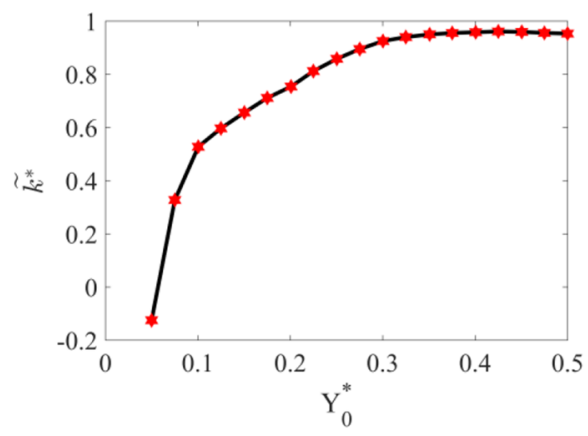

(d)
FIG. 4. Results of lock-in forced oscillation with different amplitudes at $f_{c}^{*}=f_{v}^{*}=0.177$
When $f_{c}^{*}=f_{v}^{*}=0.177$, simulations have been made in the range of $Y_{0}^{*}=(0.05,0.6)$. It is found that lock-in with the positive energy transfer from the cylinder to the fluid occurs when $Y_{0}^{*} \leq 0.5$. Figure 4 shows variations of (a) $C_{L 0}$, (b) $\phi$, (c) $b^{*}$, and (d) $\tilde{k}^{*}$ with $Y_{0}^{*}$ within the range of $Y_{0}^{*}=(0.05,0.5)$. It can be seen that with the increase in $Y_{0}^{*}, C_{L 0}$ always increases, while phase angle $\phi$ decreases. This monotonic variation with $Y_{0}^{*}$ is different from that in the previous case of $f_{c}^{*}=0.2$. We notice here that when $Y_{0}^{*} \rightarrow 0$ at this frequency $f_{c}^{*}=0.177, C_{L}$ is expected to trend to that of a fixed cylinder and its frequency is $f_{c}^{*}=f_{v}^{*}$. Thus, lock-in can occur at very low $Y_{0}^{*}$. However, as Fig. 4(d) shows, $\tilde{k}^{*}$ is already negative at $Y_{0}^{*}=0.05$, which suggests $\phi>90^{\circ}$. When $Y_{0}^{*}$ further decreases, $k$ in Eq. (19) can become negative, which suggests that the equivalent free motion is no longer possible. Of course, when $\phi>180^{\circ}$, $b^{*}$ is also negative, which makes the equivalent free motion even more impossible.

We run further simulations at another oscillation frequency $f_{c}^{*}=0.15<f_{v}^{*}$. It is found that there is no lock-in when $Y_{0}^{*}<0.15$ approximately. In the lock-in region, when $Y_{0}^{*}>0.4$ approximately, the energy transfer from the fluid to the cylinder is negative. Figure 5 shows the variation of (a) $C_{L 0}$, (b) $\phi$, (c) $b^{*}$, and (d) $\tilde{k}^{*}$ within the lock-in region, $Y_{0}^{*}=(0.15,0.4)$. It can be seen that with the increase in $Y_{0}^{*}, C_{L 0}$ increases and phase angle $\phi$ decreases monotonically, which is similar to that at $f_{c}^{*}=f_{v}^{*}=0.177$. However, with the increase in $Y_{0}^{*}$ within $Y_{0}^{*}=(0.15,0.4), b^{*}$ increases slightly initially before it decreases, while $\tilde{k}^{*}$ increases continuously, which is also similar to that $f_{c}^{*}=f_{v}^{*}=0.177$.

\section{Prediction and simulation of free motion of a body}

Form the discussion in Sec. III, when the damping $b^{*}$ and stiffness $k^{*}$ of the cylinder are given through Eqs. (19) and (20), its free motion will be the same as the forced motion. Here free oscillations with $\mathrm{m}^{*}=1-500, \mathrm{~b}^{*}=0-1.580$, and $\mathrm{k}^{*}=1-800$ have carried out to verify the mathematical analysis in Sec. III. A lock-in case under forced motion at $Y_{0}^{*}=0.556$ and $f_{c}^{*}=0.2$ is chosen as an example. From the results in Sec. IV B, we have $\tilde{k}^{*}=1.501$ and $b^{*}=0$. When $\mathrm{m}^{*}=1$, the value of $\mathrm{k}^{*}$ is equal to $4 \pi^{2} \mathrm{~m}^{*} \mathrm{f}_{\mathrm{c}}^{* 2}+\tilde{\mathrm{k}}^{*}=3.080$ predicted by forced results based on Eq. (19), which are used together with $\zeta=0$. Then, $\mathrm{C}_{\mathrm{L} 0}, \mathrm{Y}_{0}^{*}$, and $\mathrm{f}_{\mathrm{c}}^{*}$ are obtained by simulation for the free motion $\left(\mathrm{m}^{*}=1, \mathrm{k}^{*}=3.080, \mathrm{~b}^{*}=0\right)$, shown in Table IV. The comparison with the forced motion is also given in Table IV. It can be seen that results from the two simulations are virtually the same. This verifies the derivation in Sec. III.

Further from the discussion in Sec. III, in the equivalent free motion at given $f_{c}^{*}$ and $Y_{0}^{*}$, the choice of $b^{*}$ is fixed based on Eq. (20). However, the choice of $m^{*}$ and $k^{*}$ is not unique. If they vary based on Eq. (19), the motion of the body will be the same. Here we propose the definition of the damping ratio $\zeta_{c}=b / 2\left(m+M_{p}\right) \omega_{c}$, which is based on the motion frequency of the body $\omega_{c}$, instead of the natural frequency of the body $\omega_{n}$. The motion may then be the same for mechanical systems when (1) $\left(m^{*}+M_{p}^{*}\right) \zeta_{c}$ is the same and (2) the relationship between $m^{*}$ and $k^{*}$ satisfies $\tilde{k}^{*}=k^{*}-4 \pi^{2} m^{*} f_{c}^{* 2}$ from Eq. (19) predicted using the forced motion with given $f_{c}^{*}$. This may be verified numerically below.

In practical problems, $m^{*}$ is of order 1-10 in hydrodynamic engineering, which is often regarded as low mass, while $O\left(m^{*}\right)=100$ in aerodynamic applications, which is usually regarded as high mass. Thus, simulations are made with various body mass $m^{*}=1,5,250,500$ and the corresponding stiffness $k^{*}=3.080,9.397,396.3,791.1$ obtained from Eq. (19). Damping $b^{*}$ is equal to zero, and thus $\left(m^{*}+M_{p}^{*}\right) \zeta_{c}$ is still equal to zero. In such a case, $\tilde{k}^{*}=C_{L 0} / 2 Y_{0}^{*}$ is the same 


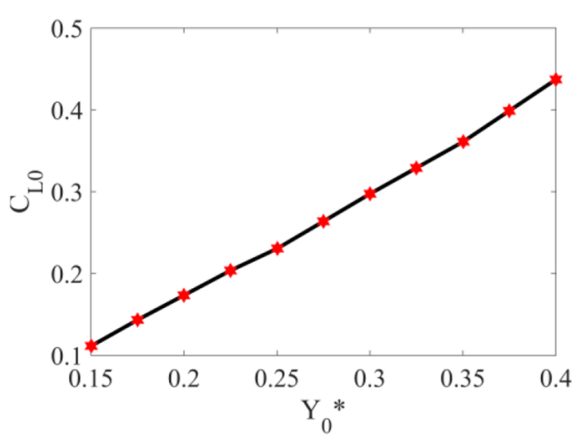

(a)

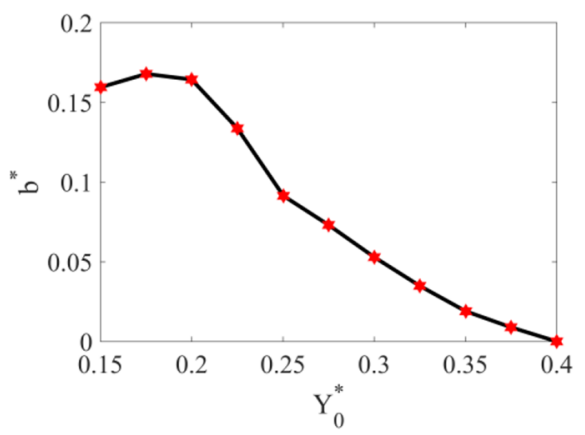

(c)

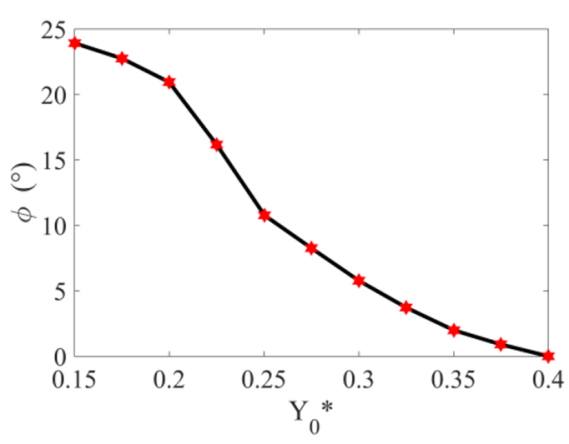

(b)

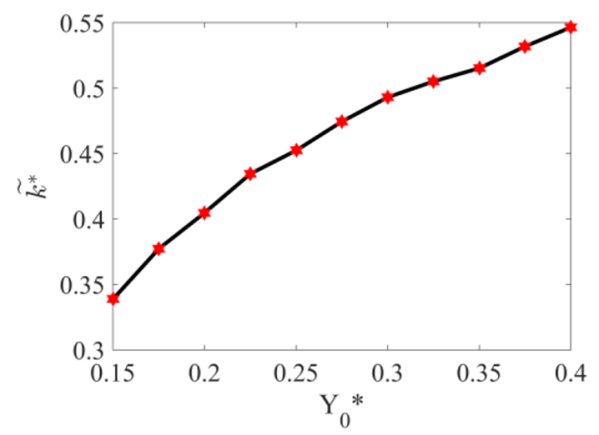

(d)
FIG. 5. Results of lock-in forced oscillation with different amplitudes at $f_{c}^{*}=0.15$. as $\tilde{k}_{0}^{*}$ adopted by Shiels et $a l{ }^{41}$ based on free motion without damping. $\mathrm{C}_{\mathrm{L} 0}, \mathrm{Y}_{0}^{*}, \mathrm{f}_{\mathrm{c}}^{*}$, and $\mathrm{f}_{\mathrm{n}}^{*}$ from simulations for free oscillations with various body masses are shown in Table IV. For $O\left(m^{*}\right)=100$, the natural frequency of cylinder $f_{n}^{*}$ is much closer to $f_{c}^{*}$ and can be regarded as $f_{n}^{*}=f_{c}^{*}$, which is similar to that under lock-in free motion for the high mass given by Feng ${ }^{27}$ and Williamson and Govardhan. ${ }^{4}$ For $O\left(m^{*}\right)=1-10$ at small mass, the natural frequency is more likely to be different from $f_{c}^{*}$. However, in these cases, it can be seen that $C_{L 0}$ and $Y_{0}^{*}$ from various body masses are virtually the same, or the motions have no difference for larger or smaller $m^{*}$.

We then consider a case with different frequency $f_{c}^{*}=f_{v}^{*}$ $=0.177$ and non-zero damping. At $Y_{0}^{*}=0.1$, we have $\tilde{k}^{*}=1.055$ and $b^{*}=1.580$ from results in Sec. IV B. Simulations are made with various body masses $m^{*}=1,5,250$, 500 , together with the structural stiffness $k^{*}=1.764,6.712$, 309.7, 618.9 based on Eq. (19). $\left(m^{*}+M_{p}^{*}\right) \zeta_{c}$ is always constant, equal to 0.639. $\mathrm{C}_{\mathrm{L} 0}, \mathrm{Y}_{0}^{*}, \mathrm{f}_{\mathrm{c}}^{*}$, and $\mathrm{f}_{\mathrm{n}}^{*}$ from simulations for free oscillations with various body masses are given in Table $\mathrm{V}$. For the high mass $O\left(m^{*}\right)=100$, the natural frequency of cylin$\operatorname{der} f_{n}^{*}$ is quite close to $f_{c}^{*}$ and it can be regarded as $f_{n}^{*}=f_{c}^{*}$, which is expected and is similar to that in the previous case

TABLE IV. Comparison of results predicted by forced oscillation $\left(\phi=0^{\circ}\right)$ with that from the real free vibration with $\left(m^{*}+M_{p}^{*}\right) \zeta_{c}=0$.

\begin{tabular}{lcccc}
\hline \hline Case & $C_{L 0}$ & $Y_{0}^{*}$ & $f_{c}^{*}$ & $f_{n}^{*}$ \\
\hline Forced motion $\left(\phi=0^{\circ}\right)$ & 1.669 & 0.556 & 0.2 & - \\
Free motion $\left(m^{*}=1, k^{*}=3.080, b^{*}=0\right)$ & 1.678 & 0.556 & 0.2 & 0.209 \\
Free motion $\left(m^{*}=5, k^{*}=9.397, b^{*}=0\right)$ & 1.694 & 0.556 & 0.2 & 0.203 \\
Free motion $\left(m^{*}=250, k^{*}=396.3, b^{*}=0\right)$ & 1.687 & 0.557 & 0.2 & 0.200 \\
Free motion $\left(m^{*}=500, k^{*}=791.1, b^{*}=0\right)$ & 1.670 & 0.556 & 0.2 & 0.200 \\
\hline \hline
\end{tabular}

in Table IV. For the low mass $O\left(m^{*}\right)=1-10$, the difference between $f_{n}^{*}$ and $f_{c}^{*}$ is larger than that with high mass. At $m^{*}=1$, the ratio of the oscillation frequency to the natural frequency of the cylinder is $f^{*} \approx 1$.2. From the table, it can be seen that $C_{L 0}$ as well as $Y_{0}^{*}$ from various body masses are virtually the same. Together with Table IV, it confirms numerically the derivation in Sec. III.

\section{D. $b^{*}-\tilde{\boldsymbol{k}}^{*}$ diagram from the forced motion predicting the free motion}

In order to predict the response of free motion based on forced motion, Staubli ${ }^{25}$ plotted the contours of $C_{L 0}$ and $\phi$ on the $f_{c}^{*}-Y_{0}^{*}$ plane and Morse and Williamson ${ }^{26}$ gave the contours of $C_{L 0} \sin \phi$ and $M_{e}^{*}$ on the $\lambda^{*}-Y_{0}^{*}$ plane, where $\lambda^{*}=1 / f_{c}^{*}$ was regarded as the nondimensionalized wavelength. For a mechanical system with given $m^{*}, k^{*}$, and $b^{*}$, its free motion results can be found from the $f_{c}^{*}-Y_{0}^{*}$ or $\lambda^{*}-Y_{0}^{*}$ plane of forced motion. In fact, at different $f_{c}^{*}$ (or $\lambda^{*}$ ) and $Y_{0}^{*}$, $C_{L 0} \sin \phi$ and $M_{e}^{*}$ can be obtained from the diagram. If the

TABLE V. Maximum lift coefficient, amplitude of the cylinder motion, oscillation frequency, and natural frequency of the cylinder under free motion at $\left(m^{*}+M_{p}^{*}\right) \zeta_{c}=0.639$ with various body masses.

\begin{tabular}{lcccc}
\hline \hline Case & $C_{L 0}$ & $Y_{0}^{*}$ & $f_{c}^{*}$ & $f_{n}^{*}$ \\
\hline $\begin{array}{l}\text { Free motion }\left(m^{*}=1,\right. \\
\left.k^{*}=1.764, b^{*}=1.580\right)\end{array}$ & 0.384 & 0.1 & 0.177 & 0.158 \\
$\begin{array}{l}\text { Free motion }\left(m^{*}=5,\right. \\
\left.k^{*}=6.712, b^{*}=1.580\right)\end{array}$ & 0.383 & 0.1 & 0.177 & 0.171 \\
$\begin{array}{l}\text { Free motion }\left(m^{*}=250,\right. \\
\left.k^{*}=309.7, b^{*}=1.580\right)\end{array}$ & 0.384 & 0.1 & 0.177 & 0.177 \\
$\begin{array}{l}\text { Free motion }\left(m^{*}=500,\right. \\
\left.k^{*}=618.9, b^{*}=1.580\right)\end{array}$ & 0.382 & 0.1 & 0.177 & 0.177 \\
\hline \hline
\end{tabular}




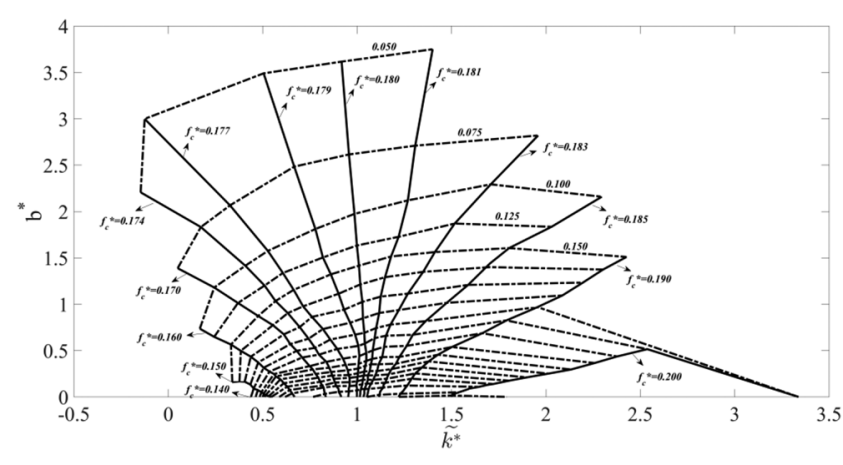

FIG. 6. $b^{*}-\tilde{k}^{*}$ diagram at various $f_{c}^{*}$ and $Y_{0}^{*}$ (solid lines denote the values of $f_{c}^{*}=0.14-0.2$ and dotted-dashed lines denote $Y_{0}^{*}=0.050-0.55$ with 0.025 increment).

equation of $b^{*}=\frac{\lambda^{* 2} C_{L 0} \sin \phi}{8 \pi^{2} Y_{0}^{*}} \sqrt{\frac{k^{*}}{m^{*}+M_{p}^{*}}}$ is satisfied, the cylinder will then oscillate with the frequency $f_{c}^{*}$ and amplitude $Y_{0}^{*}$.

In the present paper, the $b^{*}-\tilde{k}^{*}$ diagram from the forced motion results at $Y_{0}^{*}=0.05-0.55$ and $f_{c}^{*}=0.14-0.2$ is developed. Based on Eqs. (20) and (21), at given frequency, $b^{*}$ and $\tilde{k}^{*}$ will vary with amplitude $Y_{0}^{*}$. Their results can be put on the $b^{*}-\tilde{k}^{*}$ plane to form a curve. Curves at other frequencies can also be plotted as shown in Fig. 6. Similarly, at given amplitude, $b^{*}$ and $\tilde{k}^{*}$ will vary with the oscillation frequency $f_{c}^{*}$. The contours of $Y_{0}^{*}$ on the $b^{*}-\tilde{k}^{*}$ plane can also be given in Fig. 6. It can be found from the figure that when $b^{*}$ is small, the horizontal line intersects almost all $f_{c}^{*}$ lines. It means that there is a larger range of $\tilde{k}^{*}$, in which the lock-in free motion with different frequencies may be possible. When $f_{c}^{*}<0.181$, the value of $\tilde{k}^{*}$ decreases with the increase in $b^{*}$ at a given $f_{c}^{*}$. When $f_{c}^{*}>0.181$ except $f_{c}^{*}=0.200$, the value of $\tilde{k}^{*}$ increases with $b^{*}$ at a given $f_{c}^{*}$. When $f_{c}^{*}=0.200$, the value of $\tilde{k}^{*}$ increases first and then decreases with $b^{*}$. Thus, at a given $b^{*}$, the $f_{c}^{*}=0.200$ line could be intersected twice. This implies that at two different $\tilde{k}^{*}$, the motion frequency will be the same. The values of $Y_{0}^{*}$ at these two $\tilde{k}^{*}$, however, will be different. $Y_{0}^{*}$ at the smaller $\tilde{k}^{*}$ is larger than that at the larger $\tilde{k}^{*}$. For instance, when $b^{*}=0$, values of the motion frequency at $\tilde{k}^{*}=1.501$ and 3.338 are the same, $f_{c}^{*}=0.200$, while $Y_{0}^{*}=0.556$ at $\tilde{k}^{*}=1.501$ and $Y_{0}^{*}=0.250$ at $\tilde{k}^{*}=3.338$.

The diagram in Fig. 6 can provide an effective means to predict the response of a given mechanical system. For a mechanical system with damping $b^{*}$, a horizontal line with

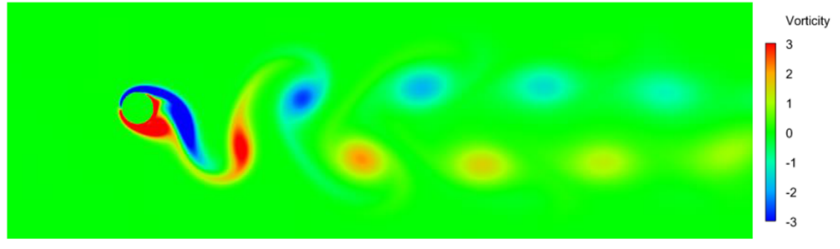

(a)

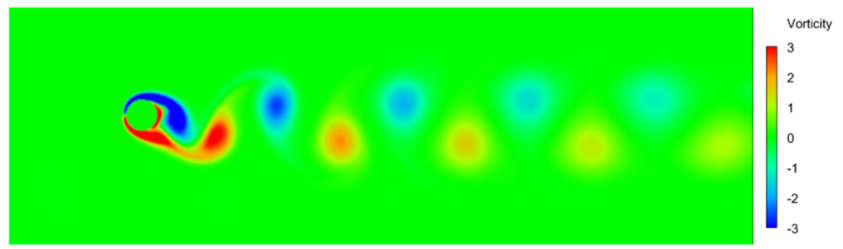

(b)

FIG. 8. Vortex contour at the maximum value of the lift coefficient for (a) point (I) and (b) point (II) shown in Fig. 7.

the same $b^{*}$ can be drawn on the $b^{*}-\tilde{k}^{*}$ plane. It will intersect the $f_{c}^{*}$ curves of different values. At each intersection of $f_{c}^{*}, \tilde{k}^{*}$ can be obtained based on this $f_{c}^{*}$, which is compared with the real one $k^{*}-4 \pi^{2} m^{*} f_{c}^{* 2}$. If they are not the same, $\tilde{k}^{*}$ will be obtained at the next $f_{c}^{*}$ and this process continues. When, at a certain $f_{c}^{*}, \tilde{k}^{*}=k^{*}-4 \pi^{2} m^{*} f_{c}^{* 2}$ is satisfied, it means that the mechanical system will oscillate with frequency $f_{c}^{*}$. In such a case, the corresponding $Y_{0}^{*}$ at this $f_{c}^{*}$ can be obtained at the point with known coordinates $\left(b^{*}, \tilde{k}^{*}\right)$ on the $b^{*}-\tilde{k}^{*}$ plane, from the $Y_{0}^{*}$ counter lines.

We may use the $b^{*}-\tilde{k}^{*}$ plane to plot curves to show how the motion of a body of mass $m^{*}$ changes with stiffness $\tilde{k}^{*}$ at different $b^{*}$. It should be noted that $Y_{0}^{*}$ and $f_{c}^{*}$ will not vary, when $m^{*}$ changes, if $\tilde{k}^{*}$ follows its change based on Eq. (19), or the free motion will be the same. We may choose $m^{*}=10$, and results are provided in Fig. 7. In some of the previous studies, the reduced velocity $U^{*}$ is often used as a horizontal axis instead of $k^{*}$, and thus it is also shown in Fig. 7. It can be seen in the figure that when $k^{*}$ increases ( $U^{*}$ decreases), $Y_{0}^{*}$ and $f_{c}^{*}$ increase first over a large range. For large $b^{*}$, they will be rising within the whole lock-in region. For small $b^{*}$, they will reach a peak and then decrease. In particular at $b^{*}=0$, they reach the peak at $k^{*} \approx 17$ and then drop sharply, which is very close to the end of the lock-in region. With a small increase in $k^{*}$, the value of $Y_{0}^{*}$ can drop from 0.556 to 0.250 and the value of $f_{c}^{*}$ can drop from 0.210 to 0.200 , which is similar to that under lock-in free motion with zero damping

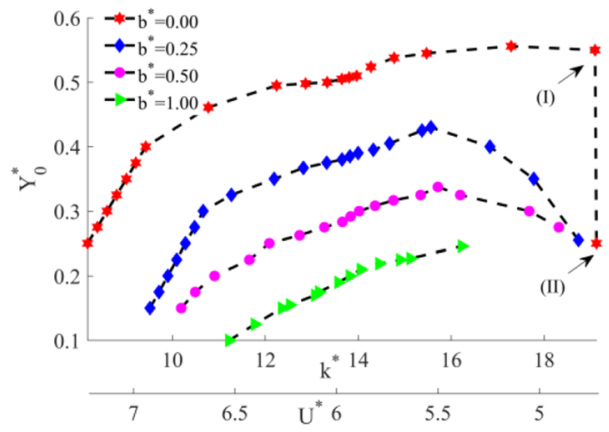

(a)

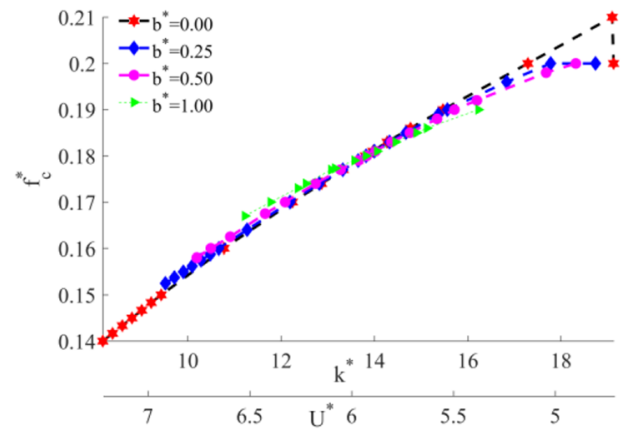

(b)
FIG. 7. Amplitude and frequency of free motion against $k^{*}$ in the lock-in region predicted from the forced motion results with $m^{*}=10$. 


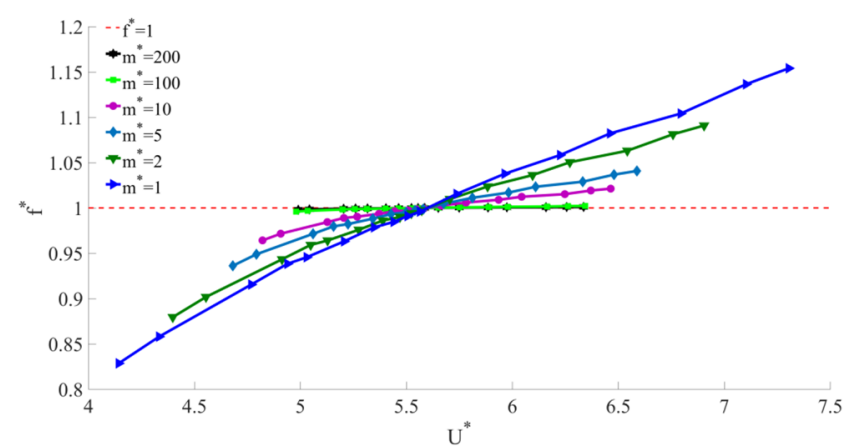

(a)

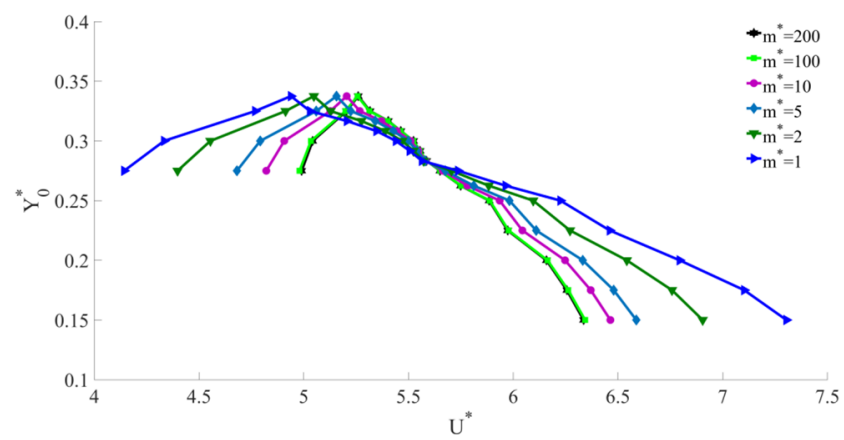

(b)

FIG. 9. The ratio between the body motion frequency and its natural frequency as well as the motion amplitude in the lock-in region predicted from the forced motion results with $b^{*}=0.5$.

in the laminar flow regime $(60<R e<200)$ given by Prasanth and Mittal. ${ }^{15}$ The main reason for this jump may be because of a change in two different wake patterns. Vortex counters at $Y_{0}^{*}=0.556\left(f_{c}^{*}=0.210\right)$ and $Y_{0}^{*}=0.250\left(f_{c}^{*}=0.200\right)$ are shown in Figs. 8(a) and 8(b), respectively. Even though these wake patterns both have a single vortex shed from each side of the cylinder during a cycle of vortex shedding, their vertical spacings of the vortices are different. In Fig. 8(a), there is a clear gap between rows, while the much smaller gap in Fig. 8(b) suggests that the effect of vortices is more like that of a single row. From Fig. 7, $Y_{0}^{*}=0.556$ is the largest possible free motion amplitude for the cylinder in the whole ranges of stiffness and damping, with the lock-in region. The corresponding motion frequency $f_{c}^{*}=0.200$, larger than $f_{v}^{*}=0.177$ of the vortex shedding frequency of a fixed cylinder.

For a mechanical system, it would also be interesting to see how the motion of the cylinder will change with $k^{*}$ for each given $m^{*}$, when $b^{*}$ is fixed. In such a case, $f_{n}^{*}=\frac{1}{2 \pi} \sqrt{\frac{k^{*}}{m^{*}+M_{p}^{*}}}$ will vary, with $k^{*}$. We notice that the range of $\tilde{k}^{*}$ for the lock-in in Fig. 6 is the same for different masses. Then, when $m^{*}$ changes from 1 to 200, the corresponding range of $k^{*}$ will also change in a similar magnitude based on Eq. (19). Thus, instead of using $k^{*}$ as a horizontal axis, we use the reduced velocity $U^{*}=1 / f_{n}^{*}$ in Fig. 9, in which the variation of $f^{*}$ and that of $Y_{0}^{*}$ with $m^{*}$ are provided. It can be seen that when $m^{*}$ is fixed, $f^{*}$ will increase with $U^{*}$. In particular, $f^{*}<1$ at smaller $U^{*}$ and $f^{*}>1$ at larger $U^{*}$. When $m^{*}$ increases, the slope of $f^{*}$ becomes milder, $f^{*}$ is closer to unity, and the largest possible free motion amplitude occurs closer to $f^{*}=1$. When $m^{*}$ reaches 100 , the slope of $f^{*}$ is almost zero and $f^{*} \approx 1$. In this sense, the peak amplitude is also at $f^{*} \approx 1$. It may also be interesting to see that with the increase in $m^{*}$, the range of $U^{*}$ becomes smaller in the lock-in region, which is similar to that observed by Govardhan and Williamson ${ }^{13}$ in free motion simulations.

Figure 7 has given the results for the whole range of stiffness at different dampings, within which lock-in occurs.

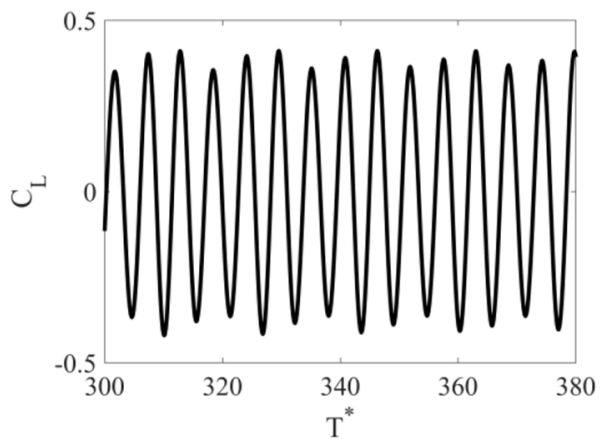

(a)

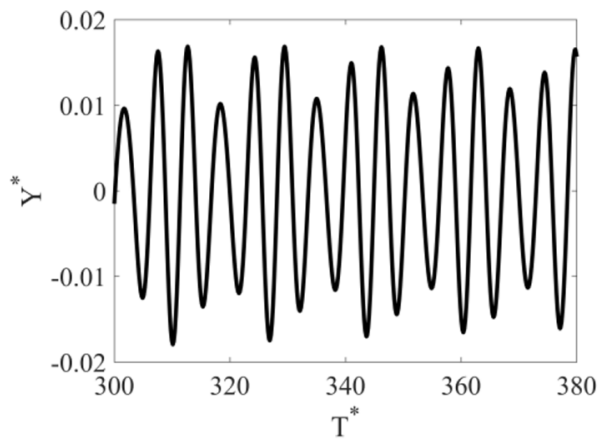

(c)

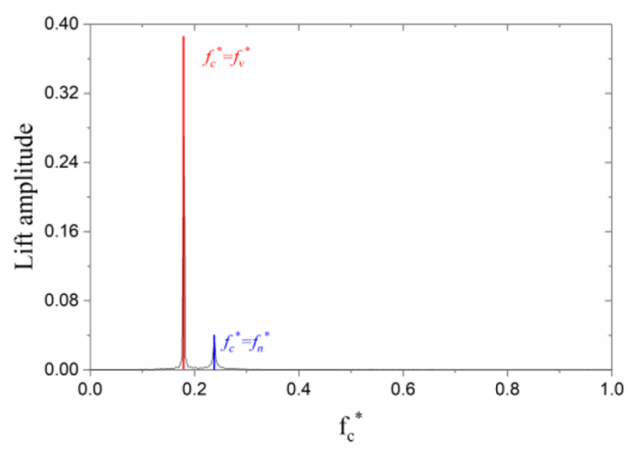

(b)

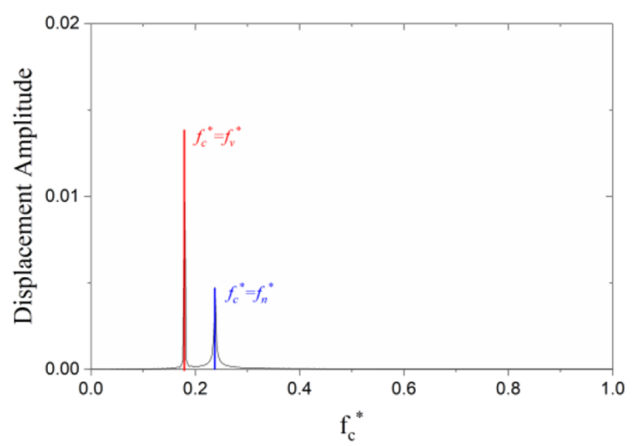

(d)
FIG. 10. Lift coefficient and displacement as well as their amplitude spectra from the free motion simulations at $m^{*}=10, b^{*}=0$, and $k^{*}=26$. 


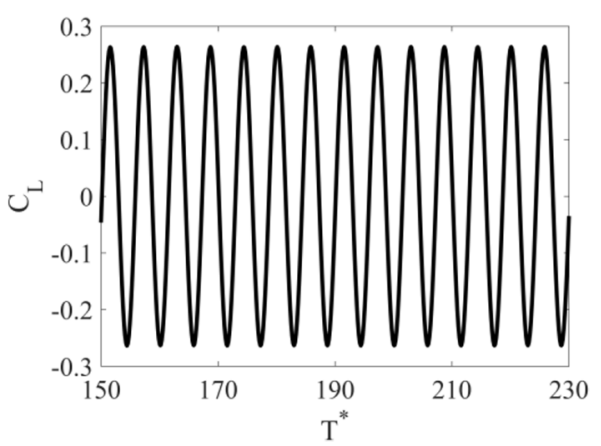

(a)

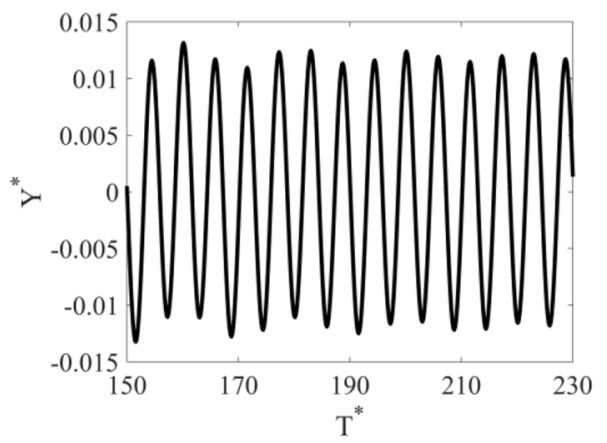

(c)

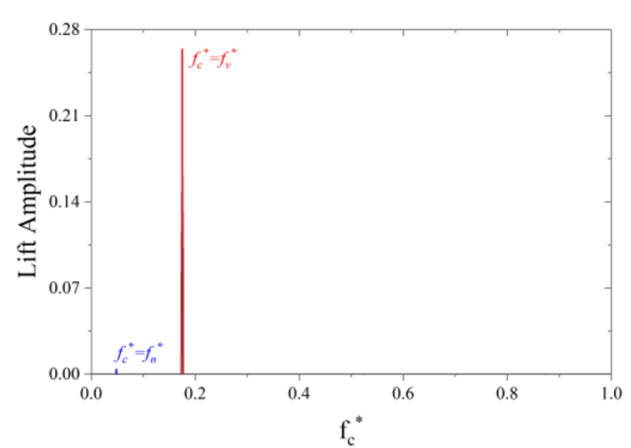

(b)

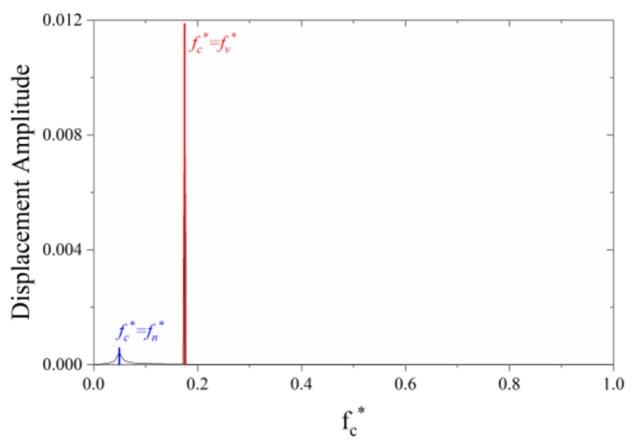

(d)
FIG. 11. Lift coefficient and displacement as well as their amplitude spectra from the free motion simulations at $m^{*}=10, b^{*}=0$, and $k^{*}=5$.
This implies that outside the range, the free motion is no longer expected to exhibit the behavior of lock-in defined in Sec. III. Thus, for $m^{*}=10$, we take a higher stiffness $k^{*}=26$ at $b^{*}=0$, which is beyond the lock-in region in Fig. 7. Figure 10 shows the lift coefficient and displacement from the free motion simulation. Their frequency spectra are also provided, similar to that in the work of Jiao and Wu. ${ }^{32}$ Similarly, results for $m^{*}=10$ and $b^{*}=0$ at lower stiffness $k^{*}=5$ outside the lock-in region are displayed in Fig. 11. We may notice that in both cases, the motion frequency has two discrete components: The one with the larger amplitude is in fact at $f_{c}^{*}=f_{v}^{*}$ and the other with the smaller amplitude is at $f_{c}^{*}=f_{n}^{*}=\frac{1}{2 \pi} \sqrt{\frac{k^{*}}{m^{*}+M_{p}^{*}}}$. This means that the cylinder is responding with oscillation at the natural frequency and at a vortex shedding frequency when it is fixed. It can be seen that $C_{L}$ and $Y^{*}$ have the same frequency components. In fact, in some previous studies on free motion, this was often regarded within the lock-in region, in which the frequencies of the lift force match those of body oscillation. ${ }^{4,32}$ Here these two cases cannot be captured by using the results in Fig. 6, as the cylinder is forced into motion at a single frequency. It is therefore important to emphasize the $b^{*}-\tilde{k}^{*}$ diagram in Fig. 6 which covers only the range of lock-in defined in Sec. III

\section{CONCLUSIONS}

The equivalence between the sinusoidal free and forced motions in the lock-in region has been established through the mathematical analysis and confirmed by the numerical results. Also, the characteristics of the free motion have been investigated based on the lock-in forced motion. From the results, the following conclusions can be drawn.
1. When a body of mass $m_{1}$, stiffness $k_{1}$, and damping $b_{1}$ is found to be in sinusoidal motion with frequency $\omega_{c}$ and amplitude $Y_{0}$, its motion will be the same at $m_{2}, k_{2}$, and $b_{2}$ if $m_{2}-m_{1}=\left(k_{2}-k_{1}\right) / \omega_{c}^{2}$ and $b_{2}=b_{1}$. This is valid even when $\omega_{c}$ is away from the natural frequency $\omega_{n}$. When $\omega_{c} / \omega_{n}=1$, this in fact reduces to a well-known result that at resonance the motion amplitude will depend on only the mass-damping parameter $\left(m+M_{p}\right) \zeta$.

2. For a body of mass $m$, added mass $M_{p}$, and damping $b$, the damping ratio may be redefined as $\zeta_{c}=b / 2(m$ $\left.+M_{p}\right) \omega_{c}$, using the motion frequency $\omega_{c}$ of the body instead of its natural frequency $\omega_{n}$. With such a definition, the sinusoidal free motion of the body depends only on the combined mass-damping parameter $\left(m+M_{p}\right) \zeta_{c}$, not on them separately. It remains to be true at both high and low mass regions provided mass changes follow the pattern in 1 above. When $\omega_{n}=\omega_{c}$, this once again becomes the existing well-known result at resonance.

3. A $b^{*}-\tilde{k}^{*}$ diagram based on the forced motion results has been established. For a body of mass $m$, stiffness $k$, and damping $b$, the diagram can be used to predict whether the body will undergo sinusoidal free motion and subsequently predict the motion amplitude and frequency.

\section{ACKNOWLEDGMENTS}

The first author is sponsored by the scholarship of China Scholarships Council, to which she is most grateful. The authors also gratefully acknowledge financial support from the Lloyd's Register Foundation (LRF) through the joint centre involving University College London, Shanghai Jiao Tong University, and Harbin Engineering University. The LRF helps 
protect life and property by supporting engineering-related education, public engagement, and the application of research.

${ }^{1}$ P. W. Bearman, "Vortex shedding from oscillating bluff bodies," Annu. Rev. Fluid Mech. 16, 195-222 (1984).

${ }^{2}$ R. D. Blevins, Flow-Induced Vibrations (Van Nostrand Reinhold, New York, 1990).

${ }^{3}$ B. M. Sumer and J. Fredsoe, Hydrodynamics Around Cylindrical Structures (World Scientific, Singapore, 1997).

${ }^{4}$ C. H. K. Williamson and R. Govardhan, "Vortex-induced vibrations," Annu. Rev. Fluid Mech. 36, 413-455 (2004).

${ }^{5}$ P. W. Bearman, "Circular cylinder wakes and vortex-induced vibrations," J. Fluids Struct. 27, 648-658 (2011).

${ }^{6}$ R. E. D. Bishop and A. Y. Hassan, "The lift and drag forces on a circular cylinder oscillating in a flowing fluid," Proc. R. Soc. A 277, 51-75 (1964).

${ }^{7}$ G. H. Koopmann, "The vortex wakes of vibrating cylinders at low Reynolds numbers," J. Fluid Mech. 28, 501-512 (1967).

${ }^{8} \mathrm{C}$. W. Van Atta and M. Gharib, "Ordered and chaotic vortex streets behind circular cylinders at low Reynolds numbers," J. Fluid Mech. 174, 113-133 (1987).

${ }^{9}$ A. Ongoren and D. Rochwell, "Flow structure from an oscillating cylinder. Part 1. Mechanisms of phase shift and recovery in the near wake," J. Fluid Mech. 191, 197-223 (1988).

${ }^{10}$ G. E. Karniadakis and G. S. Triantafyllou, "Frequency selection and asymptotic states in laminar wakes," J. Fluid Mech. 199, 441-469 (1989).

${ }^{11}$ H. M. Blackburn and R. D. Henderson, "A study of two-dimensional flow past an oscillating cylinder," J. Fluid Mech. 385, 255-286 (1999).

${ }^{12}$ K. Rayan, M. C. Thompson, and K. Hourigan, "Variation in the critical mass ratio of a freely oscillating cylinder as a function of Reynolds numbers," Phys. Fluids 17, 038106 (2005).

${ }^{13}$ R. Govardhan and C. H. K. Williamson, "Modes of vortex formation and frequency response of a freely vibrating cylinder," J. Fluid Mech. 420, 85-130 (2000).

${ }^{14}$ T. L. Morse and C. H. K. Williamson, "The effect of Reynolds number on the critical mass phenomenon in vortex-induced vibration," Phys. Fluids 21, 045105 (2009).

${ }^{15}$ T. K. Prasanth and S. Mittal, "Vortex-induced vibrations of a circular cylinder at low Reynolds numbers," J. Fluid Mech. 594, 463-491 (2008).

${ }^{16} \mathrm{M}$. Zhao and L. Cheng, "Vortex-induced vibration of a circular cylinder of finite length," Phys. Fluids 26, 015111 (2014).

${ }^{17}$ J. Kou, W. Zhang, Y. Liu, and X. Li, "The lowest Reynolds number of vortex-induced vibrations," Phys. Fluids 29, 041701 (2017).

${ }^{18}$ H. T. Ahn and Y. Kallinderis, "Strongly coupled flow/structure interactions with a geometrically conservative ale scheme on general hybrid meshes," J. Comput. Phys. 219(2), 671-696 (2006).

${ }^{19}$ I. Borazjani and F. Sotiropoulos, "Vortex-induced vibrations of two cylinders in tandem arrangement in the proximity-wake interference region," J. Fluid Mech. 621, 321-364 (2009).

${ }^{20}$ J. S. Leonitini, B. E. Stewart, M. C. Thompson, and K. Hourigan, "Predicting vortex-induced vibration from driven oscillation results," Appl. Math. Modell. 30, 1096-1102 (2006).

${ }^{21}$ S. Kumar, Navrose, and S. Mittal, "Lock-in in forced vibration of a circular cylinder," Phys. Fluids 28, 113605 (2016).
${ }^{22}$ F. S. Hover, A. H. Techet, and M. S. Triantafyllou, "Forces on oscillating uniform and tapered cylinders in cross flow," J. Fluid Mech. 363, 97-114 (1998).

${ }^{23}$ J. Carberry, R. Govardhan, J. Sheridan, D. Rockwell, and C. H. K. Williamson, "Wake states and response branches of forced and freely oscillating cylinders,” Eur. J. Mech.: B/Fluids 23, 89-97 (2004).

${ }^{24}$ J. Carberry, J. Sheridan, and D. Rockwell, "Controlled oscillations of a cylinder: Forces and wake modes," J. Fluid Mech. 538, 31-69 (2005).

${ }^{25}$ T. Staubli, "Calculation of the vibration of an elastically mounted cylinder using experimental data from forced oscillation,” J. Fluids Eng. 105, 225 (1983).

${ }^{26}$ T. L. Morse and C. H. K. Williamson, "Prediction of vortex-induced vibration response by employing controlled motion," J. Fluid Mech. 634, 5-39 (2009).

${ }^{27} \mathrm{C}$. C. Feng, "The measurements of vortex-induced effects in flow past a stationary and oscillating circular and D-section cylinders," M.Sc. thesis, The University of British Columbia, 1968.

${ }^{28}$ R. Govardhan and C. H. K. Williamson, "Defining the 'modified Griffin plot' in vortex-induced vibration: Revealing the effect of Reynolds number using controlled damping," J. Fluid Mech. 561, 147-180 (2006).

${ }^{29}$ B. J. Vickery and R. D. Watkins, "Flow-induced vibrations of cylindrical structures," in Proceedings of the First Australian Conference on Hydraulics and Fluid Mechanics, edited by R. Silvester (Pergamon Press, New York, 1964).

${ }^{30}$ R. A. Skop, "On modelling vortex-excited oscillations," NRL Memorandum Report 2927, 1974.

${ }^{31}$ A. Khalak and C. H. K. Williamson, "Motions, forces and mode transitions in vortex-induced vibrations at low mass-damping," J. Fluids Struct. 13, 813-851 (1999).

${ }^{32}$ T. Sarpkaya, "Hydrodynamic damping, flow-induced oscillations, and biharmonic response," J. Offshore Mech. Arctic Eng. 117, 232-238 (1995).

${ }^{33}$ A. Khalak and C. H. K. Williamson, "Dynamics of a hydroelastic cylinder with very low mass and damping," J. Fluids Struct. 10, 455-472 (1996).

${ }^{34} \mathrm{H}$. Jiao and G. X. Wu, "Analysis of fluctuating force acting on two cylinders in different arrangements through lattice Boltzmann method," J. Fluids Struct. 82, 101-120 (2018).

${ }^{35}$ S. Das, H. V. Patel, E. Milacic, N. G. Deen, and J. A. M. Kuipers, "Droplet spreading and capillary imbibition in a porous medium: A coupled IB-VOF method based numerical study," Phys. Fluids 30, 012112 (2018).

${ }^{36}$ C. S. Peskin, "Numerical analysis of blood flow in the heart," J. Comput. Phys. 25, 220-252 (1977).

${ }^{37}$ C. S. Peskin, "The immersed boundary method," Acta Numer. 11, 479-517 (2002).

${ }^{38}$ C. K. Aidun and J. R. Clausen, "Lattice-Boltzmann method for complex flows," Annu. Rev. Fluid Mech. 42, 439-472 (2010).

${ }^{39}$ D. A. Wolf-Gladrow, Lattice-Gas Cellular Automata and Lattice Boltzmann Models: An Introduction (Springer, New York, 2000).

${ }^{40}$ Y. H. Qian, D. d'Humieres, and P. Lallemand, "Lattice BGK models for Navier-Stokes equation," Europhys. Lett. 17(6), 479-484 (1992).

${ }^{41}$ D. Sheils, A. Leonard, and A. Roshko, "Flow-induced vibration of a circular cylinder at limiting structural parameters," J. Fluids Struct. 15, 3-21 (2001). 